\title{
Estrategias para disminuir el síndrome de Burnout en personal que labora en Instituciones de salud en Barranquilla
}

\section{Strategies to reduce Burnout syndrome in personnel working in Health Institutions in Barranquilla}

DOI: http://dx.doi.org/10.17981/cultedusoc.9.1.2018.02

Recibido: Diciembre 2 de 2017 / Aceptado: Marzo 25 de 2018

\author{
Sonia Durán ${ }^{1}$ (iD), \\ Jesús García ${ }^{2}$ iD, \\ Alejandra Parra Margel ${ }^{3}$, \\ María García Velázquez ${ }^{4}$ \\ Irmina Hernandez-Sánchez ${ }^{5}$ \\ Universidad Metropolitana de Barranquilla (Colombia) \\ sethel@unimetro.edu.co
}

Para citar este artículo

Durán, S., García, J., Parra, A., García, M. y Hernández-Sánchez, I. (2018). Estrategias para disminuir el síndrome de Burnout en personal que labora en Instituciones de salud en Barranquilla. Cultura. Educación y Sociedad 9(1), 27-44. DOI: http://dx.doi.org/10.17981/cultedusoc.9.1.2018.02

\section{Resumen}

El presente estudio se orientó a determinar estrategias de intervención para disminuir el síndrome de Burnout en personal que labora en Instituciones de salud en Barranquilla. La metodología fue de tipo descriptiva, de campo, la población fue de (26) sujetos. Se aplicó el instrumento de Maslach Burnout Inventory (MBI) constituido por 22 cuya confiabilidad es cercana al 0,9 . Se verificó que los profesionales de la salud son irrumpidos por el Síndrome de Burnout, por el régimen de trabajo estricto, se encontró que la mayoría del personal muestra un alto grado de cansancio emocional. Quienes tendrían mayor riesgo de contraer el Síndrome y por ende estar más insatisfechos laboralmente, son aquellos que tengan más de 30 años de edad, del sexo femenino.

Palabras Clave: Desgaste Profesional, Cansancio emocional, Ansiedad, estrés.

\begin{abstract}
The present study was aimed at determining intervention strategies to reduce the Burnout syndrome in personnel working in health institutions in Barranquilla. The methodology was of descriptive type, of field, the population was of (26) subjects. The Maslach Burnout Inventory (MBI) instrument was applied, consisting of 22 whose reliability is close to 0.9 . It was verified that health professionals are broken by the Burnout Syndrome, because of the strict work regime, it was found that most of the staff shows a high degree of emotional fatigue. Those who would have a higher risk of contracting the Syndrome and therefore being more dissatisfied at work, are those who are over 30 years of age, female.
\end{abstract}

Keywords: Professional Wear, Emotional Fatigue, Anxiety, Stress.

\footnotetext{
${ }^{1}$ Doctora en Ciencias Gerenciales. Docente-Investigadora de la Universidad Metropolitana de Barranquilla (Colombia).

${ }^{2}$ Doctor en Ciencias Gerenciales, Docente investigador de la Universidad Simón Bolívar de Barranquilla (Colombia).

${ }^{3}$ Doctorante en formación. Magister en Recursos humanos. Psicóloga de la Técnica Superior Universitaria en Educación Preescolar: Docente e investigadora de la Universidad de la Costa CUC de Barranquilla (Colombia).

${ }^{4}$ Doctora en Ciencias Administrativas. Profesor Investigador del Área Académica de Administración en el Instituto de Ciencias Económico Administrativas de la Universidad Autónoma del Estado de Hidalgo (México). Integrante del Cuerpo Académico en Consolidación "Gestión y Desarrollo Empresarial.

${ }^{5}$ Doctorante en formación. Magister en Psicología, Psicóloga de la Técnica Superior Universitaria en Educación Especial. Docente e investigadora de la Universidad de la Costa CUC de Barranquilla (Colombia).
}

- The author; licensee Universidad de la Costa - CUC. 


\section{Introducción}

El estado de agotamiento emocional, producido por el trabajo constituye en la actualidad un problema de salud mental que afecta a muchas personas, no obstante la dificultad existente en torno a ella es el hecho que en la mayoría de los casos, ni los afectados ni quienes los rodean reconocen a tiempo esta situación como un problema en su salud mental. Por otra parte, este es una respuesta al estrés laboral crónico integrado por actitudes y sentimientos negativos hacia las personas con las cuales se trabaja, también se asume hacia el propio rol profesional, así como por la vivencia de encontrarse emocionalmente agotado. Esta respuesta ocurre con frecuencia en los profesionales de la salud y, en general, en profesionales de organizaciones de servicios que trabajan en contacto directo con personas.

Dentro de estas perspectivas, la necesidad de estudiar el síndrome de quemarse por el trabajo en las empresas viene unida a la necesidad de estudiar los procesos de trabajo generadores de estrés laboral, así como a la inseguridad laboral y social existente en el país. Actualmente, resulta necesario considerar los aspectos de bienestar y salud laboral a la hora de evaluar la eficacia de una determinada organización. Muchas experiencias vividas en el trabajo son consideradas estresores, las cuales van acompañadas de las consiguientes reacciones crónicas de distrés, dentro de estas vivencias del trabajo existen una serie de condiciones laborales que aumentan de manera efectiva el riesgo de enfermar para los trabajadores.
De acuerdo a lo planteado, en las organizaciones se ha venido experimentando cambios de tipo tecnológico, económico y social, originados por el proceso de globalización. Ellas se han visto en la necesidad de plantearse una serie de retos para poder mantenerse como entidades competitivas dentro del mercado laboral, parte de ello es reconocer al recurso humano como fuentes de impulso propio por la significación que tiene como factor de producción. En este orden de ideas, García, Duran, Parra y Márceles (2017) manifiestan que parte de la gestión de los departamentos de recursos humanos consiste en fortalecer las condiciones laborales y personales para integrar al empleado a la organización, abarcando las características de los trabajadores tales como: habilidades, destrezas, competencias, entusiasmo y lealtad, las cuales influyen sobre los resultados, la eficiencia, la reputación y la supervivencia de la organización.

Según Herriegel (2009), esto ha inducido a generar procesos de mayor exigencia personal, promoviendo la participación de ellos en múltiples actividades formales e informales en las empresas para las cuales trabaja, además de que se produce un alto nivel de exigencia en el desarrollo de las actividades para lograr cumplir con las metas trazadas. Bajo este contexto, Duran y Parra (2016) asumen que las actividades de los individuos están enmarcadas dentro de un proceso dirigido a movilizar la energía del recurso humano, para lograr así la misión de la organización, esta situación influye en configuración de las características personales del individuo, además en cierto modo interviene en el comportamiento de los mismos sujetos. 
Dentro de este marco, la creciente globalización, conllevan un aumento de la presión ejercida sobre los trabajadores, quienes se ven envueltos entre las exigencias de las organizaciones, las demandas de los ciudadanos, sus valores y principios profesionales, por ende su familia, expresado por Duran, Prieto y García (2017), cuyas circunstancias contribuyen a la aparición del síndrome de burnout o desgaste profesional el cual ha sido objeto de múltiples investigaciones en el campo de las profesiones de servicio.

El síndrome de Burnout, para Herriegel (2009) y Gil Monte (2014), se ha definido como una respuesta al estrés laboral crónico caracterizada por el agotamiento emocional o pérdida de recursos emocionales para enfrentarse al trabajo, la despersonalización o desarrollo de actitudes de insensibilidad y cinismo hacia los receptores del servicio y la falta de realización personal o tendencia a evaluar el propio trabajo de forma negativa y la baja autoestima profesional (Maslach y Jackson: 1981,1986).

Su aparición se explica por la acción conjunta de una serie de factores antecedentes entre los que destacan los relacionados con la organización en que se desarrolla el trabajo, el tipo de tarea y los destinatarios de la acción profesional. Conlleva además una serie de consecuencias individuales (físicas, psicológicas y sociales) y organizacionales (absentismo, rotación, abandono, entre otros.). Dentro de este orden de ideas, se asume que los trabajadores hoy en día, deben esforzarse más en el desarrollo de sus actividades laborales, aportando todo el conocimiento intelectual, además de su capacidad física, expuesto por Duran y Parra (2014), Situación que indudablemente genera un proceso de estrés en el empleado originando en última instancia un desgaste emocional, conocido como desgaste profesional o síndrome de Burnout.

Efectivamente, un individuo además del estrés laboral, enfrenta el estrés de su vida cotidiana producto de los retos y problemas que se presentan en la dinámica de su vida familiar y social, exigiéndole al individuo un esfuerzo continuo de adaptación, así como de enfrentamiento. La tensión o estrés crónico generado en el ambiente de trabajo ha sido identificado como Síndrome de Burnout, tal y como lo describe Maslach tomado en (GilMonte 2007).

En este orden de ideas, el Síndrome de Burnout o desgaste profesional, también denominado síndrome de estar quemado, (Maslach y Jackson en Gil-Monte 2004), lo definen como un "síndrome de agotamiento emocional, despersonalización y desempeño personal reducido que puede ocurrir entre individuos que trabajan con gente en alguna competencia".

Dentro de este marco, Watkins (1983) citado por Gil-Monte (2007), afirma que el Burnout es un fenómeno más penetrante en el individuo que éstos últimos, implica una falta de energía y entusiasmo tanto en el hogar como en el trabajo, y puede tener un impacto devastador no sólo en la ejecución laboral sino también en las relaciones sociales y afectivas. Asimismo, Werblin (1998), planteo que el agotamiento sufrido por las personas en cuanto a su desempeño y productividad es puramente emocional, por lo cual muchos expertos dicen 
que se desarrolló como una enfermedad de la sociedad moderna, donde el trabajo deja de ser una fuente generadora de bienestar, para transformarse en un causal de desilusión. Bajo este enfoque el Burnout es un estado de agotamiento físico, emocional y mental causado por involucrarse en situaciones emocionalmente demandantes durante un tiempo prolongado.

En tal sentido, en las empresas al principio, como consecuencia de este síndrome, el empleado estaría un poco agotado. Luego, cuando la persona presenta síntomas físicos de agotamiento, se lo encontraría totalmente "oxidado" o "destruido". Pero no sólo las personas recargadas de tareas o responsabilidad son las que sufren un excesivo estrés. "Tener una vida sin motivaciones, sin proyectos, o pasar por una etapa de desocupación puede provocar el mismo desenlace" comenta el especialista. Los síntomas son iguales en ambos casos: falta de autoestima, irritabilidad, nerviosismo, insomnio y crisis de ansiedad entre otros.

De acuerdo con lo planteado, García, Duran y Prieto (2017) sostienen que en el ámbito empresarial se ha podido observar que los profesionales que ejecutan funciones de supervisión desde el aspecto administrativo, han ido adquiriendo nuevos conocimientos, competencias y experiencias a lo largo de su carrera profesional, motivados por los requerimientos del entorno social, laboral y por la búsqueda del desarrollo personal y profesional.

Actualmente en las instituciones de salud se ha podido percibir y corroborar en la dinámica diaria de los procesos ejecutados en dichas organizaciones, que el personal de supervisión y geren- cia experimentan ciertos niveles de síndrome de burnout, donde se vinculan tres dimensiones, destacando el agotamiento emocional, despersonalización y bajo logro o realización profesional o personal, entre los que se señala falta de competencia, de esfuerzo o conocimientos, al igual que carencia de expectativas y horizontes en el trabajo, asimismo una insatisfacción generalizada. Esto sustentado en Gil Monte (2011)

Al respecto, en dichas organizaciones han surgido ciertos problemas cuando los trabajadores presentan estos síntomas, ya que hay un deterioro en el rendimiento profesional y por consecuencia puede derivar a un deterioro en la calidad del servicio, absentismo laboral, alto número de rotaciones $\mathrm{y}$ abandono del puesto de trabajo, Duran, Crissien, Virviesca y García (2017) manifiestan que es importante delegar responsabilidad en los empleados para poder concentrarse en el trabajo sin tener que estar dirigiéndose continuamente a los niveles superiores dentro de la organización. De no corregir las situaciones se genera un proceso de respuesta a la experiencia cotidiana de ciertos acontecimientos, y todo ese proceso que engloba el Burnout, este varía de una persona a otra.

Entre muchas de estas organizaciones, se encuentra las Instituciones de salud, sus valores fundamentales, se conciben bajo el contexto de la ética, la excelencia, la responsabilidad, y el respeto hacia las personas hacia la empresa, orientados la búsqueda del desarrollo integral del personal, no obstante, sus objetivos se enfocan a mejorar la eficacia del sistema de gestión en salud en el área de urgencias que es el área objeto de estudio. 
Indudablemente para el cumplimiento de estos objetivos se involucran diferentes aspectos, como horarios laborales, presiones de la institución, necesidades de atención inmediata al paciente, urgencias sin controles además de diferentes elementos que se interrelacionan con los procesos de salud y necesidades del paciente, por ende, el personal responsable, debe manejar en el día a día, elementos de control mental y emocional. De acuerdo a la necesidad de cubrir los objetivos atender al paciente, se ha podido observar algunos elementos relacionados con la sobrecarga de trabajo, diferencias con el proceso de ejecución en el trabajo, faltas continuas al trabajo por parte del personal de enfermería, en algunos casos sin causa justificada.

Asimismo, la aparición de conductas que afectan el logro de los objetivos organizacionales, tales como conversaciones personales en horas de trabajo, incremento de permisos por enfermedad, además hay inconformidad por los largos períodos sin tomar vacaciones, muchas reuniones y actividades laborales los fines de semana, el problema de bajo nivel adquisitivo, además pocas o ninguna posibilidad de ascenso, Garcia, Duran, Cardeño, Prieto, Garcia y Paz (2017). En este sentido, el desgaste profesional o síndrome de Burnout ha sido reconocido por investigadores como un factor que puede afectar las actitudes personales y las condiciones de trabajo, por ende, la productividad.

No obstante, los problemas derivados por los factores que infieren en las actitudes del individuo, no es causa generada por un solo ente, es decir, por la empresa u organización, a él se le agrega una serie de situaciones personales, ambientales y ciertos elementos impulsados las necesidades personales y sociales. Dado lo anterior, los investigadores se orientaron a determinar Estrategias de intervención para disminuir el síndrome de Burnout en personal que labora en Instituciones de salud en Barranquilla.

\section{Factores que determinan la presencia del Síndrome de Burnout}

Las enfermedades relacionadas con el estrés ejercen una carga considerable sobre las personas y las organizaciones. Dentro de este marco, el Síndrome de Burnout es uno de los riesgos psicosociales emergentes en la actividad laboral se encuentra el, definido como una respuesta al estrés laboral crónico que afecta de manera especial a los profesionales de las organizaciones de servicios que trabajan en contacto con los clientes o usuarios de la organización.

Para Herriegel (2009), el síndrome de "Burnout", también llamado síndrome de "quemarse por el trabajo", de estar quemado o de desgaste profesional, se considera como la fase avanzada del estrés profesional, se produce cuando se desequilibran las expectativas en el ámbito profesional y la realidad del trabajo diario. Se define como una respuesta al estrés laboral crónico integrado por actitudes y sentimientos negativos hacia las personas con las que se trabaja, hacia el propio rol profesional, así como por la vivencia de encontrarse emocionalmente agotado. 
Actualmente, resulta necesario considerar los aspectos de bienestar y salud laboral a la hora de evaluar la eficacia de una determinada organización, pues la calidad de vida laboral, el estado de salud física y mental que conlleva tiene repercusiones sobre la institución (por ejemplo: ausentismo, rotación, disminución de la productividad, disminución de la calidad). En tal sentido, el síndrome de quemarse por el trabajo no debe identificarse con estrés psicológico, sino que debe ser entendido como una respuesta a fuentes de estrés crónico (estresores). Atendiendo a estas consideraciones, Gil-Monte y otros (1997) han señalado que esta respuesta aparece cuando fallan las estrategias de afrontamiento; este fallo supone sensación de fracaso profesional y de fracaso en las relaciones interpersonales.

Aunado a ello, el síndrome de Burnout se define según Gil-Monte (2005) como una experiencia subjetiva de carácter negativo que se caracteriza por la aparición de cogniciones, emociones $\mathrm{y}$ actitudes negativas hacia el trabajo, hacia las personas con las que se relaciona el individuo en su trabajo, en especial los clientes, y hacia el propio rol profesional. Burnout fue utilizado por primera vez por el psicólogo clínico Herbert Freudenberger para definir el desgaste extremo de un empleado.

En la actualidad es una de las causas más importante de incapacidad laboral. Su origen estaba centrado en los profesionales de la salud: los médicos. El fenómeno ha sido descrito principalmente en los profesionales de las organizaciones de servicios que trabajan en contacto directo con los clientes de esas organizaciones.
No obstante, el Burnout no está necesariamente restringido a los profesionales de los servicios humanos, pues también ha sido descrito y estudiado en trabajadores de otro tipo de organizaciones, una persona extremadamente estresada o como se diría en esta ocasión "quemada", se puede manifestar de varias formas: "el cansancio emocional es la actitud más clara, evidente y común en casi todos los casos. La persona no responde a la demanda laboral y generalmente se encuentra irritable y deprimida". También existen otras actitudes un poco más difusas. Los profesionales o empleados-enfermeras, médicos, maestros: supervisores y directivos, vendedores, entrenadores y deportistas, voluntariado, e incluso amas de casa (Gil-Monte, 2007).

De igual manera, Maslach y Jackson (1979) lo definen como un síndrome de agotamiento emocional y de cinismo que ocurre con frecuencia en los individuos que trabajan hacia personas, y que dedican mucho tiempo a las relaciones personales de carácter confidencial con esas personas bajo condiciones crónicas de estrés y de tensión.

Según Gil-Monte (2005) por agotamiento emocional se entiende la situación en la que los trabajadores perciben que ya no pueden dar más de sí mismos a nivel afectivo, donde presentan agotamiento de la energía o los recursos emocionales propios. Además, que se visualiza cuando aparece una disminución y/ o pérdida de los recursos emocionales. Existe la tendencia de los profesionales a evaluarse negativamente, de forma especial esa evaluación negativa afecta a la habilidad en la 
realización del trabajo, a la relación con las personas a las que atienden. Los trabajadores se sienten descontentos consigo mismo e insatisfechos con sus resultados laborales, este agotamiento según Gil-Monte (2005) se ve representado por medio de los indicadores en la figura 1.

Según el autor la despersonalización puede ser comprendida como el desarrollo gradual de actitudes y sentimientos negativos hacia las personas destinatarias del trabajo de forma deshumanizada debido a un endurecimiento afectivo, lo que conlleva a la generación de sentimientos de culpa frente a sus problemas. También se le llama deshumanización, porque en ella aparecen actitudes negativas, de insensibilidad.

De igual manera, la Falta de Realización personal en el trabajo se define como la tendencia de los profesionales a evaluarse negativamente, y de forma especial esa evaluación negativa afecta su capacidad para realizar el trabajo y ara relacionarse con las personas a las que atienden (por ejemplo, clientes y familiares). Los trabajadores se sienten descontentos consigo mismos e insatisfechos con los resultados laborales, suele evaluarse al trabajo de forma negativa, con vivencias de insuficiencia profesional y baja autoestima personal. Bajo este enfoque la falta

\section{Pérdida de energía}

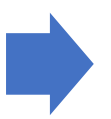

Desgaste físico

Sensación de sobre-esfuerzo

Fig. 1. Indicadores de agotamiento

Fuente: Adaptado de Gil-Monte (2005)

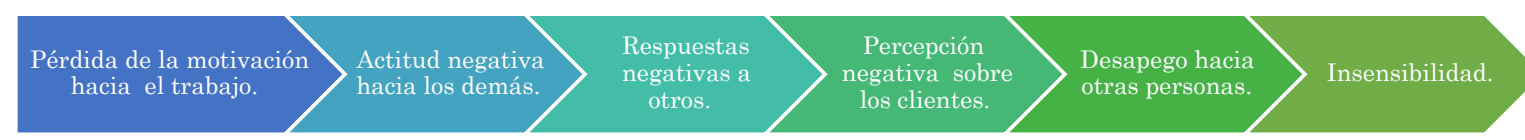

Fig. 2. Indicadores de despersonalización

Fuente: Adaptado de Gil-Monte (2005)
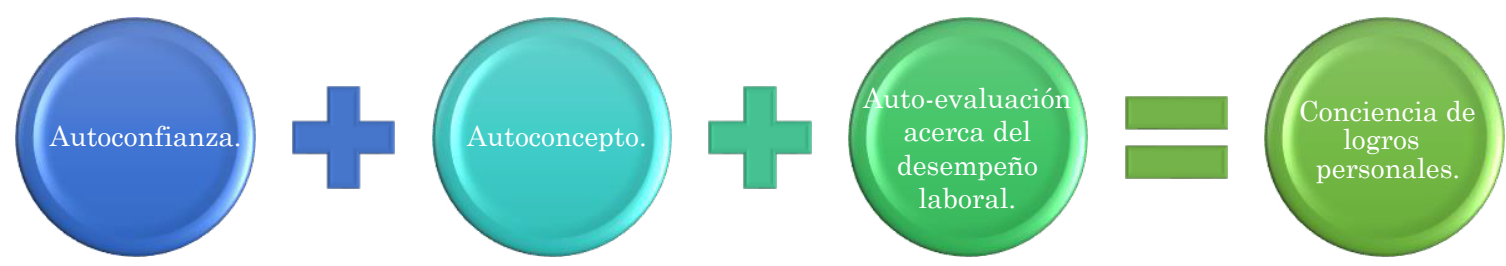

Fig. 3. Indicadores de falta de Realización personal Fuente: Adaptado de Gil-Monte (2005) 
de realización personal puede ser medida a través de los indicadores de la figura 3.

En otro orden de ideas, López (1996) presenta una serie de factores relacionados con la presencia del Síndrome de Burnout y los divide en factores individuales y factores organizacionales, donde, los primeros se refieren a patologías médicas como problemas o trastornos psicológicos, refiere que normalmente las personas se acercan al sistema de salud por estos motivos, por ejemplo, jaquecas, dolores de cabeza, úlceras, alergias, depresiones, fobias, entre otras. El sistema de Salud cuenta con remedios eficaces para esta sintomatología. Sin embargo, los otros factores que intervienen en el síndrome se les escapan, porque entre otras instituciones de salud no son de su responsabilidad. Así que el individuo estará "curado" de sus síntomas, pero las causas que lo provocan y mantienen seguirán ahí.

En segundo término, se presentan los factores organizacionales donde se ponen de manifiesto problemas estructurales o de funcionamiento en el trabajo como sobrecarga, ambigüedad y conflicto de rol, falta de supervisión, responsabilidad, inexistencia de planes de carrera, falta de apoyo social y humor, entre otros. Por todo lo dicho, se puede expresar que en la presencia del síndrome de Burnout existen una serie de factores personales y organizacionales, los cuales se encuentran presentes, pero de diferente manera en lo planteado por ambos autores.

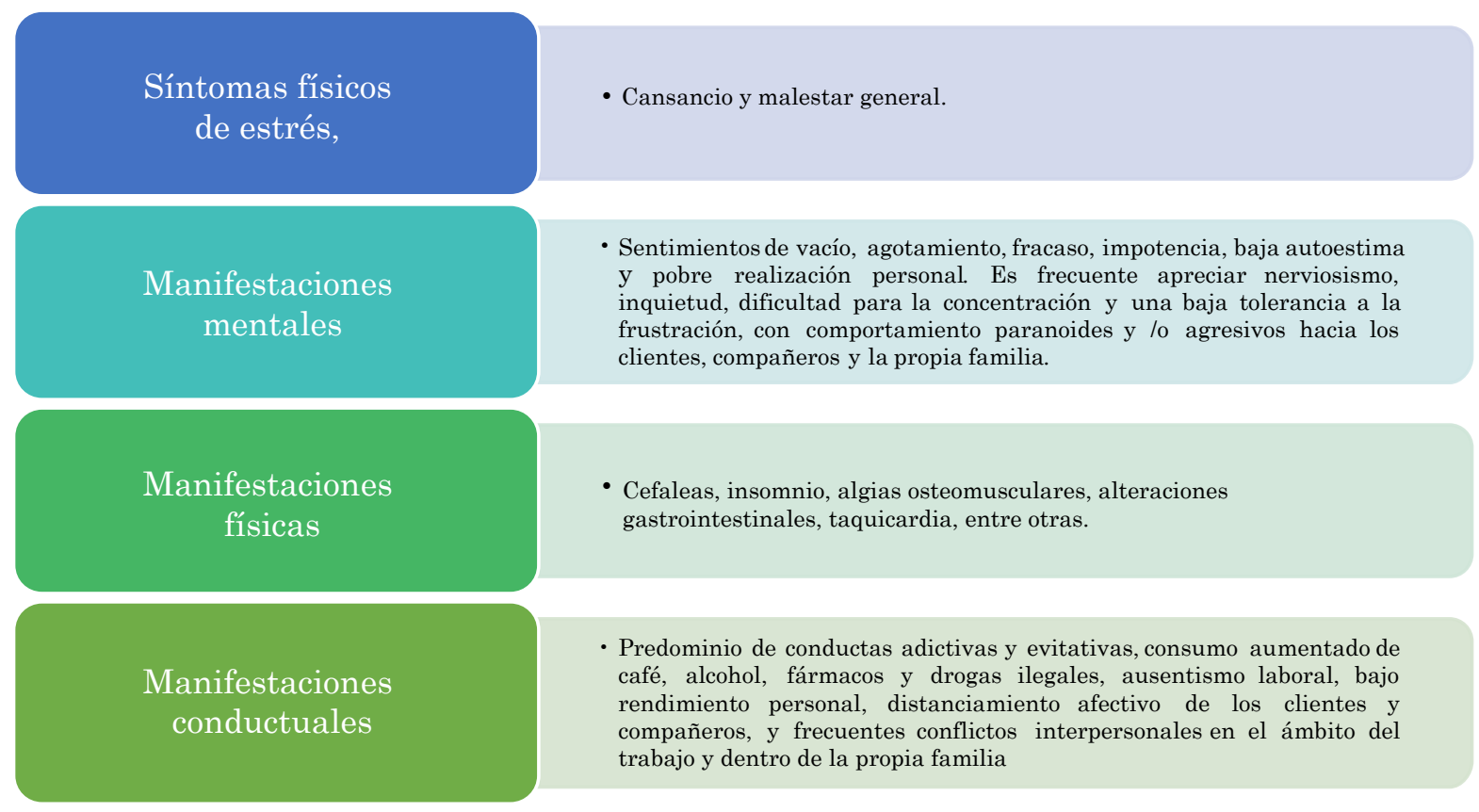

Fig. 4. Síntomas y manifestaciones asociadas Fuente: Gil Monte (2005) 
En esta situación, la respuesta desarrollada son sentimientos de baja realización personal en el trabajo y de agotamiento emocional. Ante estos sentimientos el individuo desarrolla actitudes y conductas de despersonalización como una nueva forma de afrontamiento. Así, el síndrome de quemarse por el trabajo es un paso intermedio en la relación estrés - consecuencias del estrés de forma que, si permanece a lo largo del tiempo, el estrés laboral tendrá consecuencias nocivas para el individuo, en forma de enfermedad o falta de salud con alteraciones psicosomáticas, Rozo
(2007). De acuerdo a lo expuesto, se han detectado además situaciones asociadas a los síntomas asociados.

Adicionalmente, el síndrome viene acompañado de diferentes procesos, los cuales se asumen como parte causal del problema, o quizás sea una consecuencia, en todo caso, las personas tienden a asumir actitudes y conductas que se diferencias de las habituales y que atentan contra sus emociones y la de quienes les rodean. En este orden de ideas, Gil Monte (2012) considera algunos elementos y se presentan en la figura 5 .

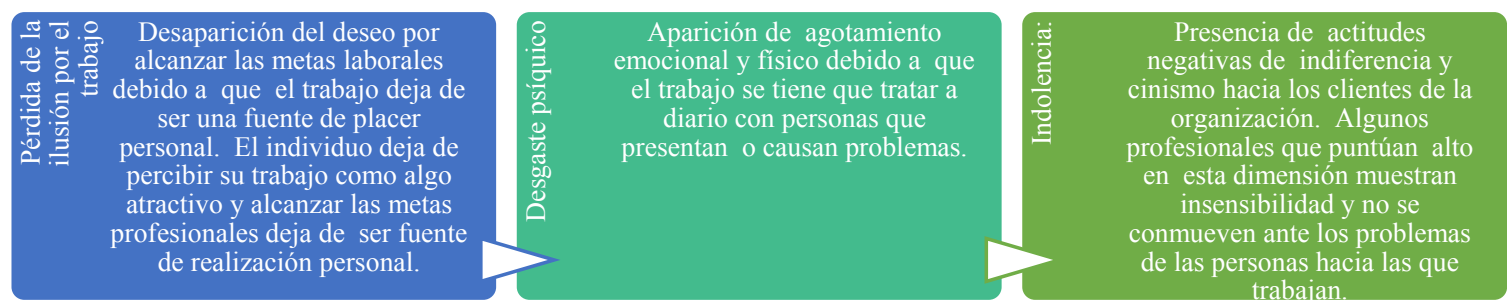

Fig. 5. Consecuencias del Sindrome de Burnout Fuente: Gil Monte (2012)

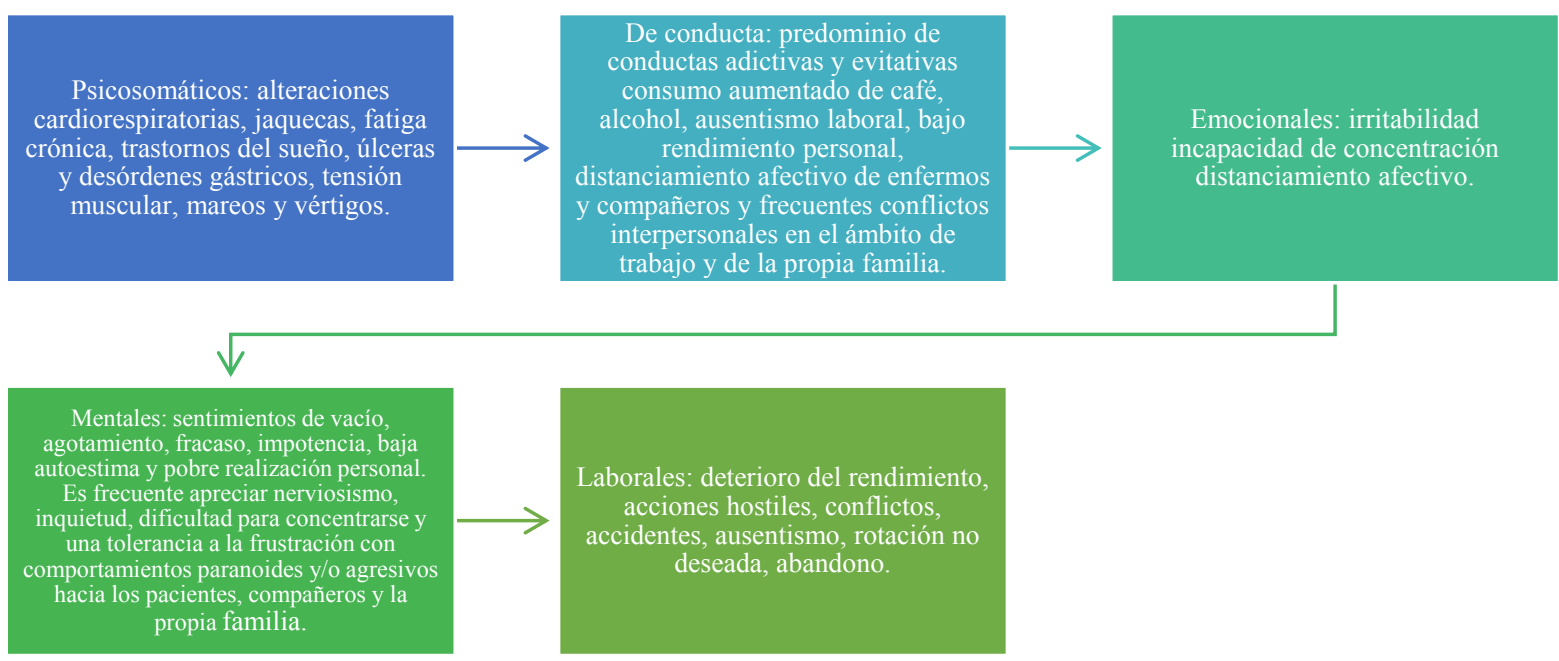

Fig. 6. Manifestaciones psicosomáticas, conductuales, emocionales, cognitivas y laborales Fuente: Gil Monte (2012) 


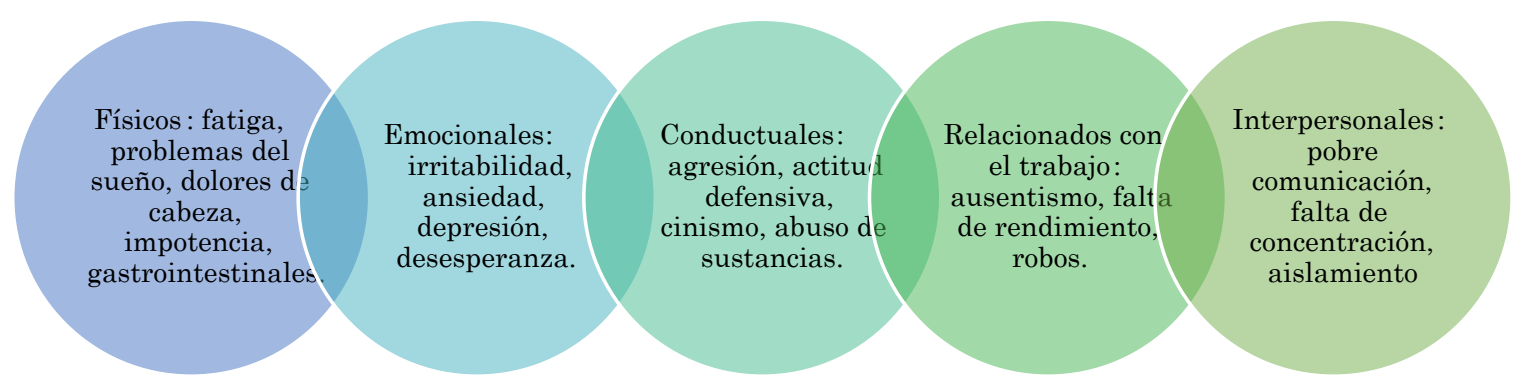

Fig. 7. Síntomas asociados multidimensionalmente con el cuadro

Fuente: López (2001).
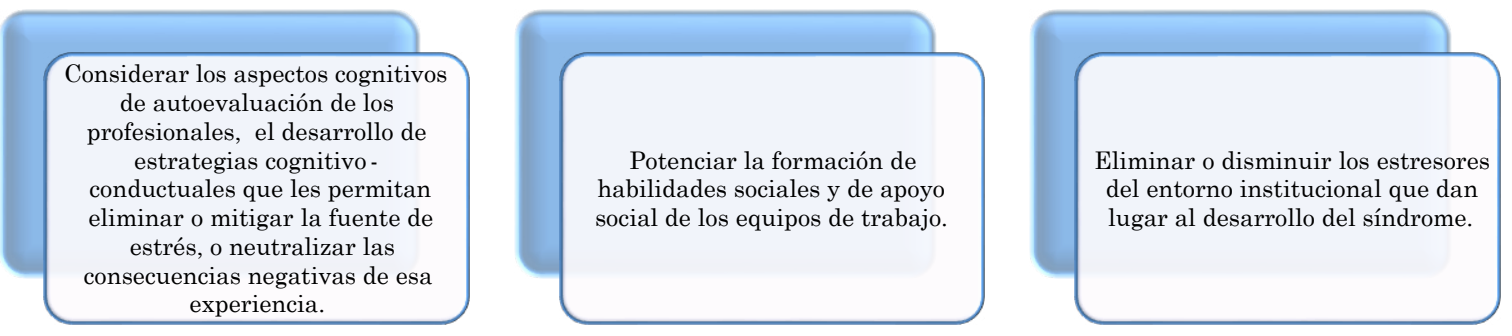

Fig. 8. Recomendaciones para la intervención del síndrome

Fuente: Cáceres (2006)

Por otra parte, López (2001) sustenta que existen otros síntomas asociados, los cuales, en un sentido más general pueden ampliar el análisis multidimensional del cuadro como se ve en la figura 7 .

En este ámbito es importante destacar lo propuesto por Leiter y Dura (1994), quienes señalaron que el síndrome de quemarse por el trabajo era fundamentalmente un constructo social que surgió como consecuencia de las relaciones interpersonales e institucionales, mientras que la depresión es un conjunto de emociones y cogniciones que tiene consecuencias sobre esas relaciones interpersonales.

\section{Estrategias de intervención del Síndrome de Burnout}

Ha quedado establecido que el síndrome de quemarse por el trabajo, es un proceso que se desarrolla como respuesta al estrés laboral crónico, de acuerdo con ello Phillips (1984) y posteriormente Cáceres (2006), sostienen que la primera medida para evitar el síndrome es conocer sus manifestaciones, además de considerar programas que implican la adquisición de conocimientos para disminuirlos, los intentos de intervención deben contemplar las siguientes recomendaciones ( $\mathrm{Fi}$ gura 8). 
Cabe destacar que la mayoría de las estrategias que abordan su prevención, recomiendan fomentar el apoyo social por parte de los compañeros. A través del apoyo social en el trabajo, las personas obtienen nueva información, adquieren nuevas habilidades o mejoran las que ya poseen, obtienen refuerzo social y retroinformación sobre la ejecución de las tareas y consiguen apoyo emocional, consejos, $\mathrm{u}$ otros tipos de ayuda. El apoyo social en el trabajo aparece en los estudios sobre el estrés laboral, además el síndrome de quemarse como una de las principales variables que permiten prevenir y aliviar las respuestas que la persona genera ante los estresores y sus efectos.

Por otro lado, en la prevención del síndrome las empresas deben desarrollar programas de prevención dirigidos a mejorar el ambiente y el clima institucional. Como parte de estos programas se sugiere desarrollar programas de socialización anticipatoria lo cual ha sido desarrollado por Duran (2010) con el objetivo de acercar a los profesionales a la realidad laboral y evitar el choque con sus expectativas irreales. Se recomienda también, desarrollar los procesos de retroinformación grupal e interpersonal desde los compañeros.
En el mismo sentido, Herriegel (2009) considera que los programas organizacionales e individuales para ayudar a los gerentes y empleados a enfrentar el estrés se han vuelto cada vez más populares, conforme se sabe más de las impresionantes consecuencias del estrés. Existen diversos métodos para que los individuos y las organizaciones manejen el estrés y reduzcan sus efectos dañinos. La intervención se asume de la siguiente manera (Figura 9).

En este sentido, las intervenciones constituyen para las empresas una inversión tanto de carácter humano y social, como laboral y económico. En convergencia con los aportes de Peña (2015) y Oblitas, et al. (2017) referentes al diseño de modelos de intervención organizacional que incrementen los niveles de bienestar laboral y calidad de los colaboradores.

\section{Método}

La metodología del estudio fue de tipo descriptiva, de corte transaccional y la muestra de estudio estuvo conformada con (26) participantes. Se aplicó el instrumento de Maslach Burnout Inventory (MBI) constituido por 22 cuya confiabilidad fue (a: 0,9).

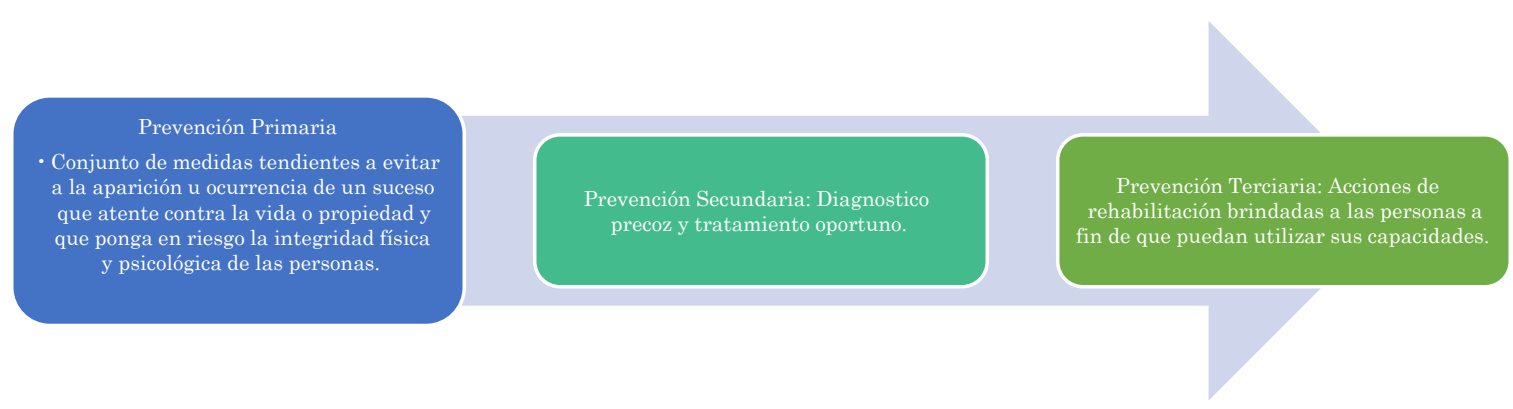

Fig. 9. Niveles de prevención primaria, secundaria y terciaria Fuente: Herriegel (2009) 


\section{Participantes}

Respecto a la población, está conformada por los (26) empleados quienes ejercen labores de supervisión y gerencia en instituciones de salud, bajo este contexto, el personal incluido dentro del contexto del universo a estudiar presenta un carácter finito, determinado y accesible de manejar, cuya identificación y afiliación institucional se reservan por motivos de confidencialidad y consentimiento informado.

Tabla 1.

Caracterización de la Muestra de Estudio

\begin{tabular}{cc}
\hline Nivel de Cargo & Participantes \\
\hline Gerente medico & 1 \\
Jefe de emergencia & 2 \\
Jefe de cirugía & 2 \\
Jefe de enfermera & 3 \\
Médicos de urgencias & 6 \\
Personal de enfermería & 12 \\
\hline Muestra Total & 26 \\
\hline
\end{tabular}

Fuente: Elaboración Propia (2018)

\section{Instrumento}

En este estudio, de acuerdo con la clasificación metodológica, se utilizó la técnica de observación mediante encuesta, caracterizada por la facilidad en su aplicación, orientación e interpretación, atendiendo al hecho de que su orientación corresponde con las características referidas sobre la población. De esta manera se aplicó el Maslach Burnout Inventory (MBI) de Maslach y Jackson, constituido por 22 ítems en el cual se le plantea al sujeto una serie de enunciados sobre sus sentimientos y pensamientos en relación a diversos aspectos de su interacción continua con el trabajo y su desempeño habitual. Cabe destacar que este instrumento tiene una alta consistencia interna y una confiabilidad ( $\alpha: 0,9)$. Cabe resaltar que el instrumento es un cuestionario auto-administrado, donde el sujeto ha de contestar a cada enunciado a partir de la pregunta mediante una escala tipo Likert.

\section{Procedimiento}

En primera instancia se seleccionó la Organización de Salud cuya identificación se reserva por motivos de confidencialidad. Posteriormente, se estableció el proceso de solicitud de autorización organizacional y el consentimiento informado para cada uno de los participantes, La tabulación se llevó a cabo con la finalidad de organizar la información obtenida en las encuestas aplicadas en el estudio. Por tanto, requiere la realización de un proceso sistemático y cuidadoso en relación al traslado de las respuestas emitidas por cada sujeto de la muestra a una tabla de tabulación. En el presente estudio se utilizó una matriz de doble entrada para procesar los datos obtenidos en la encuesta aplicada al personal Una vez aplicado los instrumentos los mismos se procesaron, mediante la utilización del programa SPSS Versión 22.0.

En razón a que el estudio es de tipo descriptivo, se ejecutó un tratamiento estadístico de igual naturaleza. A tal fin consistió en el cálculo de la distribución de frecuencias absolutas y frecuencias relativas (\%) de las respuestas arrojadas por la población de estudio. Estas frecuencias se representaron en tablas de doble entrada y gráficos.

\section{Resultados}


El deterioro emocional puede ser grave cuando este tiene sobrecarga física y psíquica a causa de las muchas horas de trabajo, dedicación, presiones y tensiones en la empresa. De esta manera los estresores laborales generaran una serie de estrategias de afrontamiento, en el caso de los profesionales cuyo objeto de trabajo son personas, deben ser efectivas para manejar las respuestas al estrés, pero también han de ser eficaces para eliminar los estresores. A continuación, se presentan resultados asociados al tema en estudio.

Tabla 1.

Motivación para la realización de las tareas

\begin{tabular}{ccc}
\hline Alternativas & Frecuencia & $\mathbf{\%}$ \\
\hline Siempre & 20 & 77 \\
Casi Siempre & 4 & 15 \\
A Veces & 1 & 4 \\
Casi Nunca & 1 & 4 \\
Nunca & 0 & 0 \\
Total & 26 & 100 \\
\hline
\end{tabular}

Fuente: Elaboración Propia (2018)

A los empleados se les preguntó, si sentían pocas ganas de Realizar su Tarea a lo cual respondieron en un $77 \%$ que siempre les pasa, mientras un 15\% afirma que casi siempre están en esa posición, el $4 \%$ opino algunas veces, y el otro 4\% consideró que nunca tiene esas sensaciones. Por lo que se concluye que en la empresa el personal administrativo la mayoría de las veces siente pocas ganas de realizar su tarea.

En cuanto al Cansancio al realizar sus tareas los empleados se manifestaron en un $77 \%$ que siempre se sienten cansados, un $15 \%$ informa que casi siempre está cansado, un 4\% alega que algunas veces, otro $4 \%$ manifestó la opción casi nunca, esto indica el personal de supervisión y gerencia se siente cansado al realizar sus tareas.

Tabla 2.

Cansancio al realizar las tareas

\begin{tabular}{ccc}
\hline Alternativas & Frecuencia & $\mathbf{\%}$ \\
\hline Siempre & 20 & 77 \\
Casi Siempre & 4 & 15 \\
Algunas Veces & 1 & 4 \\
Casi Nunca & 1 & 4 \\
Nunca & 0 & 0 \\
Total: & & 100 \\
\hline
\end{tabular}

Fuente: Elaboración Propia, (2018)

Tabla 3.

Desinterés por el trabajo

\begin{tabular}{ccc}
\hline Alternativas & Frecuencia & $\%$ \\
\hline Siempre & 17 & 65 \\
Casi Siempre & 5 & 20 \\
Algunas Veces & 4 & 15 \\
Casi Nunca & 0 & 0 \\
Nunca & 0 & 0 \\
Total: & 26 & 100 \\
\hline
\end{tabular}

Fuente: Elaboración Propia, (2018)

A continuación, se analiza la posición del empleado con respecto al sentir poco interés por su trabajo, a lo que el 65\% respondió a la alternativa siempre, mientras que un 20\% alegó que casi siempre sien te poco interés por su trabajo, un $16 \%$ algunas veces, por lo que se determina como factor a ser considerado la situación expresada con respecto al no sentir interés para realizar el trabajo, situación que induce a disminuir los niveles de rendimiento.

Tabla 4. 
Actitudes agresivas y hostiles

\begin{tabular}{ccc}
\hline Alternativas & Frecuencia & \% \\
\hline Siempre & 16 & 63 \\
Casi Siempre & 7 & 27 \\
Algunas Veces & 2 & 8 \\
Casi Nunca & 0 & 0 \\
Nunca & 1 & 4 \\
Total: & 26 & 100 \\
\hline
\end{tabular}

Fuente: Elaboración Propia, (2018)

A los empleados se les preguntó si habían notado que sus actitudes hacia los otros se habían vuelto hostiles, estos respondieron en un $62 \%$ que siempre estaba pasando, mientras que en un $27 \%$ opinaron que casi siempre, el 8\% algunas veces, mientras solo el $4 \%$ concluyo que nunca eso le ocurría. No obstante, la mayoría del personal sostiene que está teniendo actitudes hostiles hacia sus compañeros de trabajo, ello podría repercutir hacia el cliente.

Tabla 5.

Problemas y alteraciones del sueño

\begin{tabular}{|c|c|c|}
\hline Alternativas & Frecuencia & $\%$ \\
\hline Siempre & 21 & 80 \\
\hline Casi Siempre & 2 & 8 \\
\hline Algunas Veces & 1 & 4 \\
\hline Casi Nunca & 2 & 8 \\
\hline Nunca & 0 & 0 \\
\hline Total: & 26 & 100 \\
\hline
\end{tabular}

Fuente: Elaboración Propia, (2018)

En lo concerniente a los Problemas para conciliar el sueño, la gran mayoría de los encuestados representados por un $80 \%$ respondió que siempre tenían estos problemas, el 8\% respondió casi siempre, un $4 \%$ algunas veces, el $8 \%$ restante con- sidera que nunca ha tenido problemas para conciliar el sueño. S3e concluye que la mayoría del personal encuestado ha tenido problemas para conciliar el sueño, generándole cansancio físico y emocional.

Tabla 6.

Manifestaciones de ansiedad

\begin{tabular}{ccc}
\hline Alternativas & Frecuencia & $\mathbf{\%}$ \\
\hline Siempre & 21 & 80 \\
Casi Siempre & 2 & 8 \\
A Veces & 1 & 4 \\
Casi Nunca & 2 & 8 \\
Nunca & 0 & 0 \\
Total: & 26 & 100 \\
\hline
\end{tabular}

Fuente: Elaboración Propia, (2018)

Con respecto a si el empleado siente mucha ansiedad, la población opino en un $80 \%$ que siempre le ocurre esa situación, el 8\% alega que casi siempre, un $4 \%$ algunas veces, el $8 \%$ restante opina que casi nunca siente mucha ansiedad. Los resultados indican que el personal de supervisión y gerencia siente mucha ansiedad en cuanto al desarrollo de sus procesos de trabajo.

Tabla 7.

Migrañas y cefaleas

\begin{tabular}{ccc}
\hline Alternativas & Frecuencia & \% \\
\hline Siempre & 24 & 92 \\
Casi Siempre & 0 & 0 \\
Algunas Veces & 1 & 4 \\
Casi Nunca & 1 & 4 \\
Nunca & 0 & 0 \\
Total: & 26 & 100 \\
\hline
\end{tabular}

Fuente: Elaboración Propia, (2018)

En lo referente a si padece de dolores de cabeza, se determinó que un $92 \%$ de la 
población encuestada manifestó que siempre sentía dolores de cabeza, un $4 \%$ opino que casi siempre, y otro 4\% respondió casi nunca, Esto refiere que el personal administrativo de Instituciones de salud integrante de la Población objeto de estudio, padece continuamente de dolores de cabeza, lo cual podría estar ocasionado por desgaste profesional.

Tabla 8.

Vacio Emocional

\begin{tabular}{ccc}
\hline Alternativas & Frecuencia & $\%$ \\
\hline Siempre & 23 & 88 \\
Casi Siempre & 2 & 8 \\
Algunas Veces & 1 & 4 \\
Casi Nunca & 0 & 0 \\
Nunca & 0 & 0 \\
Total: & 26 & 100 \\
\hline
\end{tabular}

Fuente: Elaboración Propia, (2018)

En cuanto a la tabla y grafico 8, las respuestas obtenidas fueron las siguientes, un $88 \%$ de la población manifiesta que siempre Al terminar su día de trabajo, siente vacío emocionalmente, un $8 \%$ opino que casi siempre le ocurría, mientras un $4 \%$ alego que algunas veces sentía esa sensación, esto refleja que dicha situación agota al empleado de tal forma de generarle desgastes emocional y físico.

\section{Conclusiones}

Se verificó que los profesionales de la salud son irrumpidos por el Síndrome de Burnout, dado como un régimen de trabajo estricto, con carga horaria semanal de hasta 40 horas, sin tiempo para formación, incurriendo en disminución de su calidad de vida. En virtud de que el síndrome nace cuando se reconoce que el trabajo, como escenario social-laboral provoca múltiples fuentes de estresores, este llega a afectar a la gente perjudicando la atención al paciente, al afectar a la salud física y mental de los trabajadores.

Dentro de las áreas de mayor deterioro en relación al Síndrome, se encontró que la mayoría del personal muestra un alto grado de cansancio emocional. Quienes tendrían mayor riesgo de contraer el Síndrome y por ende estar más insatisfechos laboralmente, son aquellos que tengan más de 30 años de edad, del sexo femenino.

Contribuye al incremento de riesgos de accidentes, Puede afectar el rendimiento y ausentismo laboral, Puede crear un clima psicosocial insano en la organización laboral, Puede ser un factor de riesgo de Enfermedad, Puede ser un factor de riesgo de incapacidad laboral por alteraciones somáticas y/o psicológicas. A partir de estos planteamientos, se sugieren ejecutar algunos lineamientos como agentes de prevención:

- Escuchar a la persona de forma activa, pero sin darle consejos ni juzgar sus acciones.

- Dar apoyo técnico en el sentido de que un compañero experto le confirme que está haciendo bien las instituciones de salud.

- Crear en el trabajador necesidades y pensamientos de creatividad, desafío, e implicación en el trabajo por parte de aquellos compañeros expertos. Estos compañeros deben ser competentes en su profesión

- Apoyo emocional entendido como apoyo incondicional. Este tipo de apoyo es necesario para todas las personas y si no es posible obtenerlo en el trabajo la persona debe disponer de él en casa.

- Apoyo emocional desafiante, que 
haga reflexionar a la persona sobre si realmente ha agotado todas las posibles soluciones, que le haga replantearse las posibles atribuciones hechas sobre los resultados de la tarea (culpar a los compañeros o superiores de los fracasos profesionales).

- Participar en la realidad social de la persona, confirmando o cuestionándole las creencias sobre sí mismo, sobre su autoconcepto, autoeficacia y autoestima.

Se considera que en la prevención del síndrome la institución debe desarrollar programas de prevención dirigidos a mejorar el ambiente y el clima institucional. Como parte de estos programas se sugiere desarrollar programas de socialización anticipatoria con el objetivo de acercar a los profesionales a la realidad laboral y evitar el choque con sus expectativas irreales. Se recomienda, desarrollar los procesos de retroinformación grupal e interpersonal desde los compañeros. El objetivo de estos programas se centra en mejorar el ambiente y el clima institucional mediante el desarrollo de equipos de trabajo eficaces

Recomendaciones de la Organización internacional del trabajo para diagnosticar situaciones de desgaste profesional en la empresa

- Realizar Diagnósticos de las fuentes y niveles de estrés

- Realizar una planificación preventiva de manera de reducir o evitar estresores que pueden ser causas de demandas ambientales

- Poner en marcha programas de prevención e intervención sobre el estrés en aquellas áreas o grupos de personas de mayor riesgo.

- Evaluación de los programas para ver su eficacia y realizar cambios si fuera necesario

\section{Referencias}

Duran, S. E. (2010). Diversidad Cultural y Socialización Organizacional del personal administrativo de las universidades privadas del municipio Maracaibo. Universidad Dr. Rafael Belloso Chacín. [Trabajo de grado para optar al título de Doctor]. En Ciencias Gerenciales. Maracaibo. Venezuela.

Duran, S. E., \& Parra M. (2016). Condiciones Laborales y Calidad de vida en el trabajo. Estrategias innovadoras: Afrontando los retos de la economía global., 1, 437-459. Universidad autónoma del Estado de Hidalgo y Universidad Autónoma de Tlaxcala.

Duran, S., \& Parra, M. (2014). Diversidad Cultural para promover el desarrollo de habilidades sociales en educación superior. Cultura, Educación y Sociedad, 5(1), 5567. Recuperado de: http://revistascientificas.cuc.edu.co/index.php/ culturaeducacionysociedad/article/ view/995

Duran, S., García, J., Crissien, J., \& Virviesca, J. (2017). Estrategias gerenciales para la formación de equipos de trabajo en empresas constructoras del Caribe Colombiano. Espacios, 38(13), 26-28. Recuperado de: http://www.revistaespacios. com/a17v38n13/a17v38n13p24.pdf.

Duran, S.E., García, J., \& Prieto, R. (2017). Influencia de la Calidad de Vida en el rendimiento del estudiante universitario. Caracas: Fondo Editorial UNERMB. 
Garcia J, Duran S, Cardeño E, Prieto R, Garcia E \& Paz A. (2017) Proceso de planificación estratégica: Etapas ejecutadas en pequeñas y medianas empresas para optimizar la competitividad. Espacios, 38(52), 16-30. http://www.revistaespacios. com/a17v38n52/17385216.html

García, J. E., Durán, S. E., Parra, M. A., \& Márceles, V. S. (2017). Dirección estratégica del talento humano para el fomento de valores en los cuerpos policiales venezolanos. $E s$ pacios, 38(32), 16-28. Recuperado de: http://www.revistaespacios.com/ a17v38n32/a17v38n32p16.pdf.

Gil-Monte, P. R. (2012). The influence of guilt on the relationship between burnout and depression. European Psychologist [Online].

Gil-Monte, P.R., y Peiró, J.M. (1999). Validez factorial del Maslach Burnout Inventory en una muestra multiocupacional. Psicothema, 11, 679- 689.

Grau-Alberola, E., Gil-Monte, P.R., García-Juesas. J.A., y FigueiredoFerraz, H. (2010). Incidence of burnout in Spanish nursing professionals: A longitudinal study. International Journal of Nursing Studies, 47, 1013-1020.

Herriegel, D., \& Slocum, J. (2009). Comportamiento Organizacional, 10 ed. México: Editorial Thompson.

Leda, R., Palomera, A. \& Morante, M. (2007). El síndrome de burnout en profesionales de enfermería mexicanos. En P. Gil-Monte y B. Moreno-Jiménez. El Síndrome de Quemarse por Trabajo (Burnout). Grupo profesionales de riesgo. Madrid: Pirámide.
López, J. J. (1996). El desgaste ocupacional en el personal sanitario que trabaja con enfermos afectados por el virus de la inmunodeficiencia humana. Folia Neuropsiquiatría, 31, 39- 55

López, J. M. O., Santiago, M. J., Godás, A., Castro, C., Villardefrancos, E., \& Ponte, D. (2008). An integrative approach to burnout in secondary school teachers: Examining the role of student disruptive behaviour and disciplinary issues. International Journal of Psychology and Psychological Therapy, 8(2), 259-270.

Maslach, C, \& Jackson, S. (1986). Maslach Burnout Inventory. Palo Alto: Consulting Pysichologists Press.

Maslach, C., Schaufeli, B., \& Leiter, M. (2001). Job Burnout. Annou Rev Pshycol., 52, 397-422.

Oblitas-Guadalupe, L. A., TurbayMiranda, R., Soto-Prada, K. J., Crissien-Borrero, T., Cortes-Peña, O. F., Puello-Scarpati, M., \& UcrósCampo, M. M. (2017). Incidence of Mindfulness and Qi Gong on the State of Health, Psychological WellBeing, Life Satisfaction and Work Stress. Revista Colombiana de Psicología, 26(1), 99-113.

Peña, O. F. C. (2015). Well-Being Labor Teaching in the Educational Public Sector of Barranquilla-Colombia. Procedia-Social and Behavioral Sciences, 191, 2835-2843.

Prieto Pulido, R. A., Villasmil, M., \& Urdaneta, L. (2011). Gestión humana en organizaciones postmodernas. Base fundamental hacia la excelencia organizacional. $\mathrm{Ci}$ cag, 8(2), 1-12. 
Prieto, R., Burgos, C., García, J., \& Rincón, Y. (2016). Mercadeo interno para optimizar la calidad de servicio en la banca universal. Revista Venezolana de Gerencia, 21(73).
Rozo, M. (2007). Evaluación del síndrome de estrés asistencial en los profesionales de la salud en una institución hospitalaria de III nivel en la ciudad de Bogotá. Psychologia: Avances en la Disciplina 2007; 1(1), 185-214 